American Journal of Microbiology and Immunology
(ISSN: 2474-2910)

\title{
High Prevalence of Drug Resistant Neisseria gonorrhoeae in Males with Urethral Discharge at the STD Clinic Mulago Hospital Uganda
}

\author{
George Ndawula ${ }^{1 *}$, Henry Kajumbula ${ }^{1}$, Fred Nsubuga ${ }^{3}$, Edgar Kigozi $^{1}$, Freddie Bwanga ${ }^{1,2}$ \\ ${ }^{1}$ Department of Medical Microbiology Makerere University College of Health Sciences, ${ }^{2} \mathrm{MBN}$ \\ Clinical Laboratories, ${ }^{3}$ Ministry of Health.
}

\section{ABSTRACT}

Among the treatable sexually transmitted infections (STIs), Neisseria gonorrhoeae is considered to be most important because of its ever evolving antibiotic resistance. Currently third generation cephalosporins are recommended as first-line drugs in many countries. Unfortunately, resistance to these cephalosporins (cefixime and ceftriaxone) has been reported elsewhere. The objectives of the study were to determine the prevalence of Neisseria gonorrhoea in adult males presenting to Mulago hospital with urethral discharge and to determine the level of resistance of Neisseria gonorrhea to the commonly available antimicrobial agents at the clinic focusing on extended-spectrum cephalosporins. In a cross sectional study, we enrolled 112 adult males aged 18-65 yrs with urethral discharge at the STD clinic Mulago Hospital. Prevalence of gonorrhoea was determined based on Gram staining and/or N. gonorrhea isolation at culture. Antimicrobial susceptibility testing was determined with the Kirby-Bauer disk diffusion method and with the Nitrocefin tests for $\beta$-lactamase production. Among 112 adult male patients aged 18-65 years with urethral discharge,the prevalence of $\mathrm{N}$. gonorrhea was at $81.3 \%$. Resistance to drugs was identified at the following rates: tetracycline $100 \%$, ciprofloxacin $94.8 \%$, cotrimoxazole $91.4 \%$, penicillin $82.8 \%$, cefixime $20.7 \%$, ceftriaxone $5.2 \%$, spectinomycin $1.7 \%$, and azithromycin $0 \%$. According to this study drug resistance to commonly available antimicrobials for $\mathrm{N}$. gonorrhea is very high among isolates from patients attending the STI clinic, Mulago Hospital Kampala, Uganda. Ceftriaxone, spectinomycin and azithromycin appeared to be the most useful drugs in the treatment of $\mathrm{N}$. gonorrhea infection at the clinic. This work was completed in November 2017.

Keywords: Antimicrobial resistance, cefixime, ceftriaxone, cephalosporins, Neisseria gonorrhea.
*Correspondence to Author:

George Ndawula

Department of Medical Microbiology Makerere University College of Health Sciences

\section{How to cite this article:}

George Ndawula, Henry Kajumbula, Fred Nsubuga, Edgar Kigozi, Freddie Bwanga.High Prevalence of Drug Resistant Neisseria gonorrhoeae in Males with Urethral Discharge at the STD Clinic Mulago Hospital Uganda. American Journal of Microbiology and Immunology, 2022, 7:11.

\section{eScîPub}

eSciPub LLC, Houston, TX USA. Website: http://escipub.com/ 


\section{INTRODUCTION}

Neisseria gonorrhoea is the bacterium [1] that causes gonorrhea a major reproductive health problem. Gonorrhoea follows Chlamydia as the most prevalent sexually transmitted bacterial diseases in humans. Globally the World Health Organization in 2011 estimated that about 88 million new cases of gonococcal infections occurred in 2005 . Around $95 \%$ of infected males are symptomatic ${ }^{[2]}$. An estimated $10 \%-20 \%$ of infected women develop the following complications [2] ; Pelvic inflammatory disease, ectopic pregnancy, infertility, and adverse pregnancy conditions. Males develop, testicular, prostate infections and infertility while young ones develop Neonatal ophthalmia and blindness. It is also a co-factor in increasing HIV[ by 500 fold] ${ }^{[3]}$ and HPV infections; this could be due to viral load increase in the semen or vaginal fluids of those co-infected with gonorrhea and HIV and HPV, and to an increased number of inflammatory cells for HIV and HPV [4-6].The gonococci produces only mildly symptomatic or asymptomatic disease in females while around $95 \%$ of infected males are symptomatic. Therefore, in the absence of a vaccine, appropriate diagnostics and antimicrobial treatments are the key elements for the reduction and control of gonorrhea and its associated complications. About 30-60 patients with STIs visit the STI clinic daily for treatment in Mulago hospital. A major factor to the continued spread of gonococcal infections is the ability of $N$. gonorrhoea to acquire resistance to antibiotics; over the last two decades, genes which encode for resistance in $N$. gonorrhoea strains to antimicrobial agents like; Spectinomycin, penicillin, tetracycline and, quinolones have been characterized in different countries ${ }^{[7-9]}$

The mechanisms for chromosomally mediated resistance to penicillin $\mathrm{G}$ and cephalosporin in $N$. gonorrhoea involve the penA, penB, and $m$ trR mutations. Mutations in penA causes insertion of a single amino acid into penicillin-binding protein 2 [PBP 2], and this reduces the level of binding of penicillin to PBP 2.[10-11]. The penB mutation, which is a mutation that is linked to the porin gene, reduces porin permeability to hydrophilic antibiotics and plays an important role in the development of resistance to penicillin $G$, ${ }^{[12]}$. Mtr gene mutations increase the level of expression of the MtrCDE efflux pump and confers resistance to multiple hydrophobic agents and some hydrophilic antibiotics such as the penicillins [13]. The reduced susceptibility of $N$. gonorrhoea strains to broad spectrum cephalosporins such as cefixime and ceftriaxone has been proposed to be associated with polymorphisms in several of these genes and especially with certain penA mosaic alleles $^{[14]}$.These mosaic sequences are thought to have evolved from recombination events involving pen $A$ gene sequences from several Neisseria species, including $N$. perflava, $N$. sicca, and $N$. cinerea ${ }^{[14-15]}$.

Before 2010, treatment failures had only been reported in Japan , ${ }^{[16]}$ however, two cases of clinical failures with cefixime standard treatment were described in 2010 in Norway ${ }^{[17]}$, and three cases in 2011 in the United Kingdom ${ }^{[18-19]}$. In July 2011 the first Neisseria gonorrhea strain with resistance to cefixime was found in Austria $^{[20]}$. Furthermore, the first gonococcal strain with high-level clinical resistance to ceftriaxone [the last remaining option for empirical first-line treatment], was recently found in Japan ${ }^{[21]}$. In Uganda studies done in Mulago hospital STI clinic indicate that gonorrhea cases are increasing which may be due to; prevalence of resistant strains in communities, and improper treatment guidelines.

There is limited data on the extended spectrum cephalosporin resistance among Neisseria gonorrhea strains in Uganda. This resistance may be chromosomal or plasmid encoded. This study therefore, intended to determine the prevalence of cefixime and ceftriaxone resisitant strains and to determine other conventional drug susceptibility patterns among Nesseria gonorrhoea strains isolated from adult 
males presenting to Mulago hospital with urethral discharge.

\section{Materials and Methods}

A cross sectional study to identify Neisseria gonorrhoea from urethral discharge samples collected from men was done. Participants aged $18 y$ rs to 65yrs were recruited from sexually transmitted infections [STI] clinic at Mulago Hospital [Uganda] .This hospital admits patients from all corners of the country and its clinic is a national referral centre for patients with STIs. The Clinical Microbiology Laboratory, Molecular Biology Laboratory [Makerere University] and MBN Laboratories did the laboratory work.

Identification of $\boldsymbol{N}$. gonorrhea: 112 samples were collected, Gram stained, cultured on a selective modified Thayer martin agar under 5$7 \% \quad \mathrm{CO}_{2}$ and the following phenotypic characteristics tests; colony morphology, catalase , superoxol, glucose utilization ,colistin and oxidase tests performed. After performing those phenotypic tests, Prevalence was determined using the following procedures ; Gram negative intracellular diplococci and culture negative, Gram negative intracellular diplococci and culture positive, No primary Gram reaction, culture positive with extracellular diplococci on a slide. And negative results were determined by obtaining no morphotypes suggestive of neisseria on gram reaction and culture plate. All cultured samples, which were positive for $N$. gonorrhoea, were tested using the Vitek 2 system for definitive identification according to manufacturer's instructions.

\section{This study used a control strain of neisseria gonorrhoea no. ATCC43069}

For storage; about 3-5 colonies of each pure isolate was inoculated into a cryovial containing $0.5-1.0 \mathrm{ml}$ of $20 \%$ glycerol in trypticase soy broth and stored at $-80{ }^{\circ} \mathrm{C}$

Antimicrobial susceptibility testing; This was performed by the Kirby-Bauer disk diffusion method to obtain the zones of inhibition for the susceptibility of 11 antibiotics as summarized in table 1 below. Briefly, an overnight growth of each isolate was suspended in Muller-Hinton Broth and the suspension adjusted to $0.5 \mathrm{Mc}$ Farland standard .This was used to inoculate plates of GC agar base with Growth factor mixture GFM 80005. Antibiotic disks were then placed onto the inoculated plates which were then incubated at $37^{\circ} \mathrm{C}$ for 24 hours. Zone diameters were then measured and interpretation made using CLSI break points as seen in the table below.

Table 1 ; Drug susceptibility break points as recommended by CLSI [22]

\begin{tabular}{|c|c|c|c|c|}
\hline DRUGS & DISC POTENCY & SUSCEPTIBLE & INTERMEDIATE & RESISTANCE \\
\hline Ceftriaxone & $30 \mu \mathrm{g}$ & $39-51 \mathrm{~mm}$ & ---- & $\leq 39 \mathrm{~mm}$ \\
\hline Cefixime & $5 \mu g$ & $\geq 37 \mathrm{~mm}$ & & $\leq 37$ \\
\hline Penicillin & 10 units & $\geq 47 \mathrm{~mm}$ & $27-46 \mathrm{~mm}$ & $\leq 26 \mathrm{~mm}$ \\
\hline Ciprofloxacin & $5 \mu g$ & $\geq 41 \mathrm{~mm}$ & $28-40 \mathrm{~mm}$ & $\leq 27 \mathrm{~mm}$ \\
\hline Tetracycline & $30 \mu \mathrm{g}$ & $\geq 38 \mathrm{~mm}$ & $31-37 \mathrm{~mm}$ & $\leq 30 \mathrm{~mm}$ \\
\hline Azithromycin & $15 \mu \mathrm{g}$ & $\geq 42 \mathrm{~mm}$ & ------ & $\leq 19 \mathrm{~mm}$ \\
\hline Spectinomycin & $100 \mu \mathrm{g}$ & $\geq 18 \mathrm{~mm}$ & $15-17 \mathrm{~mm}$ & $\leq 14 \mathrm{~mm}$ \\
\hline Doxycycline & $30 \mu \mathrm{g}$ & ND & ND & ND \\
\hline Erythromycin & $15 \mu \mathrm{g}$ & ND & ND & ND \\
\hline Septrin & $1.25 / 23.5 \mu \mathrm{g}$ & $\geq 16 \mathrm{~mm}$ & $11-15 \mathrm{~mm}$ & $\leq 10 \mathrm{~mm}$ \\
\hline Gentamycin & $120 \mu \mathrm{g}$ & ND & ND & ND \\
\hline
\end{tabular}

ND- These ranges have not been determined yet by CLSI 
Beta-lactamase production; Nitrocefin strips were used to detect beta-lactamase production and double disc diffusion tests were also performed to detect extended beta -lactamase producing strains using Cefixime and Augmentin discs plated $30 \mathrm{~mm}$ apart from the center of each other on GC media .

\section{RESULTS}

\section{Prevalence of gonorrhea determined by gram staining or culture .}

Of the 112 participants 91 had gram stains and positive cultures suggestive of Neisseria giving a prevalence of $81.3 \%$.

Urethral discharge for the ages of 18- 30 years , 31-43yrs and 44-65yrs were $68.7 \%, 27,6 \%$ and $3.5 \%$ respectively, a total of $65[58 \%]$ were single and 47 [42\%] were married . A total of 47 [42\%] admitted to having used an antibiotic while the rest $65[58 \%]$ denied history of antibiotic use to treat their urethral condition.

\section{Drug susceptibility profiles}

Table 2 below summarizes the drug susceptibility profiles of 11 antibiotics.

Resistances for; ceftriaxone, cefixime, azithromycin ,tetracycline ,ciprofloxacin, septrin , spectinomycin and penicillin was identified at the following rates $5.2 \%, 20.7 \%, 0 \%, 100 \%$, $94.8 \%, 91.4 \%, 1.7 \%$ and $82.8 \%$ respectively.

Gentamycin, doxycycline and erythromycin are commonly used in Uganda however, their standard zones of susceptibility among $N$. gonorrhea strains have not yet been determined by CLSI today and we were not able to put them in any of our susceptibility categories.

However, gentamycin had 36 zones of inhibition ranging from $16 \mathrm{~mm}-30 \mathrm{~mm}, 22$ zones of inhibition ranging from $30-60 \mathrm{~mm}$,

Doxycycline had all its zones below $20 \mathrm{~mm}$. and erythromycin had 10 of its zones of inhibition below $22 \mathrm{~mm}$

\section{TABLE 2: \% DRUG SUSCEPTIBILITY PROFILES FOR FIFTY EIGHT ISOLATES [positive at culture]}

\begin{tabular}{|l|l|l|l|l|}
\hline DRUGS & \%SUSCEPTIBLE & \%INTERMEDIATE & RESISTANCE & \%RESISTANCE \\
\hline Ceftriaxone & 94.8 & ---- & 3 & 5.2 \\
\hline Cefixime & 79.3 & & 12 & 20.7 \\
\hline Penicillin & 12.1 & 5.1 & 48 & 82.8 \\
\hline Ciprofloxacin & 0 & 5.2 & 55 & 94.8 \\
\hline Tetracycline & & 0 & 58 & 100 \\
\hline Azithromycin & 100 & ----- & 0 & 0 \\
\hline spectinomycin & 98.3 & 0 & 1 & 1.7 \\
\hline Doxycycline & ND & ND & N D & \\
\hline Erythromycin & ND & ND & ND & \\
\hline Septrin & 3.4 & 5.2 & 53 & 91.4 \\
\hline Gentamycin & ND & ND & ND & \\
\hline
\end{tabular}

ND- These ranges have not yet been determined by CLSI.

\section{Discussions}

The recent emergence and transmission of Neisseria gonorrhoeae strains with reduced susceptibility to expanded-spectrum cephalosporins such as cefixime and ceftriaxone have been reported. Effective control of the disease spread requires ; improved knowledge about the prevalence of the disease, timely and appropriate diagnosis and treatment coupled with increased disease awareness promotion. 
The aim of this study was to determine the prevalence of the gonococcus in adult males with urethral discharge and to determine the drug susceptibility profiles of $N$. gonorrhoeae to cefixime and ceftriaxone drugs .

\section{Prevalence of Neisseria}

112 participants were enrolled for the study. The prevalence of the gonococcus disease in adult males presenting with urethral discharge was found at $81.3 \%$, this is slightly lower than a $95 \%$ prevalence found In the UK, by a study done on diagnosis rates for gonorrhoea in 2008 [2]. Another study done in Bangui on aetiology of uretrhal discharge ${ }^{[23]}$ found a $65 \%$ prevalence of the gonococcus in urethral discharges . while a study done on antimicrobial susceptibility survey of $N$, gonorrhoea in a Uganda ubarn clinic by Kamya et al [ 1989-1993] reported a prevalence of $73 \%$ and one done recently in Uganda on prevalence ,socio-demographic factors and antimicrobial susceptibility of Neisseria gonorrhea found a prevalence of gonorrhea at $55 \%$ [24].

These differences could be attributed to the different methods used to collect and to transport urethral samples coupled with the different identification techniques used.

In this study the prevalence is higher than the ever stated one in Uganda, this is true because we recruited male participants presenting with urethral discharge. There usually $95 \%$ chances of detecting Neisseria gonorrhoea in males with urethral discharge ${ }^{[2]}$. This prevalence may also be due to the development of resistance to several antimicrobial drugs such as sulfonamides, penicillin, tetracyclines and quinolones by $N$. gonorrhoea and lack of antimicrobial resistance [AMR] surveillance which could help to establish and maintain the efficacy of standard treatment regimens.

Nitrocefin tests were positive reflecting penicillinase production by these strains.

Findings from Urethral Discharge ; Urethral discharge was more among the ages of 18-30 years than among the 31-43yrs and 44-65yrs which had $68.7 \%, 27,6 \%$ and $3.5 \%$ respectively, a total of 65 [58\%] were single and 47 [42\%] were married, this shows that urethral discharge was high among the unmarried participants. A total of 47 [42\%] admitted to having used an antibiotic while the rest $65[58 \%]$ denied history of antibiotic use to treat their urethral condition

\section{Antimicrobial susceptibility profiles}

Cetriaxone,cefixime, gentamycin,cipropfloxacin. Spectinomycin,erythromycin. Tetracycline. Doxycycline.penicillin, azithromycin and septrin were antibiotics used to determine the susceptibility of fifty eight culture positive isolates. Ceftriaxone resistance was found at $5.2 \%$, Cefixime at $20.7 \%$, Penicillin at $82.8 \%$ while Ciprofloxacin and Tetracycline had the worst resistance rates of $94.8 \%$ and $100 \%$ respectively. All the gonococcus strains were susceptible to azithromycin and spectinomycin at rates of $100 \%$ and $98.3 \%$ respectively.

This is the first study to report ceftriaxone and cefixime resistance in Uganda. Before 2010, treatment failures had only been reported in Japan , ${ }^{16]}$, however, two cases of clinical failures with cefixime standard treatment were described in 2010 in Norwayl17], and three cases in 2011 in the United Kingdom ${ }^{[18-19]}$. In July 2011 the first Neisseria gonorrhea strain with resistance to cefixime was found in Austria ${ }^{[20]}$. Furthermore, the first gonococcal strain with high-level clinical resistance to ceftriaxone [the last remaining option for empirical first-line treatment], was recently found in Japan ${ }^{[21]}$. This study also revealed that doxycyline a drug which is also used here in Uganda with cefixime to treat Gonorrhoea had very small zones of inhibition bellow $20 \mathrm{~mm}$ and these ranges were similar to those of tetracycline a sister drug which also showed $100 \%$ resistance. Since they are both tetracyclines, the organisms may be resistant to both drugs.

Erythromycin which is also used in pregnancy to treat the gonococcus showed very small zones of inhibition [below 22mm] which don't compare with those of Azithromycin[42mm] which showed $100 \%$ susceptibility which is a 
sister drug and therefore may also be regarded as a drug of resistance.

\section{Conclusions}

Gonorrhoea remains a leading cause of urethritis among males accounting for $81.3 \%$ of cases of urethral discharge at Mulago . Resistance to $3^{\text {rd }}$ generation cephalosporins exists among Uganda $N$. gonorrhoeae strains at a rate of $20.3 \%$ for Cefixime and $5.2 \%$ for ceftriaxone among study strains . cefixime and ceftriaxone drugs are now reported as drugs of resistance in Uganda as far as Gonorrhoea treatment is concerned, however, the results cannot be generalized because the study was done in a referral hospital where a small population of patients attend for treatment. The disease was more among adult males below the age of 30years which are the more active years for the youth. Prior use of drugs affected the prevalence of the disease in that many who had the discharge failed to give positive results and the disease was more found in singles other than the married ones.

\section{Recommendations:}

Current use of ceftriaxone as a first line therapy for urethritis should continue but treatment should be prolonged, Cefixime use should be withdrawn and a final decision on its future use be made after a broader study while azithromycin and spectinomycin which showed susceptible rates at $100 \%$ and $98.3 \%$ respectively, should also be included on the treatment guidelines in Uganda. And in order to prevent future resistances, combination therapies with azithromycin and ceftriaxone should be recommended.

\section{Limitations}

The small sample size used which was due to a low number of expected cases with urethritis at the STI clinic may have affected the overall prevalence.

Participants who didn't have a clear cut discharge didn't want to give a sample because of the swabbing procedure which is slightly painful and so loss of these participants may have affected the study.

\section{Acknowledgement}

I acknowledge my supervisors who did a wonderful job to make this study successful.

Thanks go to the Makerere university microbiology and molecular laboratory staff who also made this study a success.

Special thanks go to the pharmaceutical society of Uganda who made a partial funding for this study.

And lastly special thanks also go to MBN laboratories staff who also made a contribution for the successful completion of this work.

\section{References}

[1]. Todar K. Todar's online textbook of bacteriology: University of Wisconsin-Madison Department of Bacteriology; 2006.

[2]. Moran JS. Gonorrhoea. Clinical evidence. 2007;2007.

[3]. Tapsall J, Limnios E, Murphy D. Analysis of trends in antimicrobial resistance in Neisseria gonorrhoeae isolated in Australia, 1997-2006. Journal of Antimicrobial Chemotherapy. 2008;61[1]:150-5.

[4]. Ghys PD, Fransen $K$, Diallo $M O$, EttiègneTraoré V, Coulibaly I-M, Yeboué KM, et al. The associations between cervicovaginal HIV shedding, sexually transmitted diseases and immunosuppression in female sex workers in Abidjan, Cote d'Ivoire. Aids. 1997;11[12]:F85F93.

[5]. Cohen MS, Hoffman IF, Royce RA, Kazembe P, Dyer JR, Daly CC, et al. Reduction of concentration of HIV-1 in semen after treatment of urethritis: implications for prevention of sexual transmission of HIV-1. The Lancet. 1997;349[9069]:1868-73.

[6]. Mostad SB, Overbaugh J, DeVange DM, Welch MJ, Chohan B, Mandaliya K, et al. Hormonal contraception, vitamin A deficiency, and other risk factors for shedding of HIV-1 infected cells from the cervix and vagina. The Lancet. 1997;350[9082]:922-7.

[7]. Stathi M, Flemetakis A, Miriagou V, Avgerinou $\mathrm{H}$, Kyriakis KP, Maniatis AN, et al. Antimicrobial susceptibility of Neisseria gonorrhoeae in Greece: data for the years 1994-2004. Journal of Antimicrobial Chemotherapy. 2006;57[4]:775-9. 
[8]. Wang B, Xu J-s, Wang C-x, Mi Z-h, Pu Y-p, Hui $M$, et al. Antimicrobial susceptibility of Neisseria gonorrhoeae isolated in Jiangsu Province, China, with a focus on fluoroquinolone resistance. Journal of medical microbiology. 2006;55[9]:1251-5.

[9]. Enders M, Turnwald-Maschler A, Regnath T. Antimicrobial resistance of Neisseria gonorrhoeae isolates from the Stuttgart and Heidelberg areas of southern Germany. European Journal of Clinical Microbiology and Infectious Diseases. 2006;25[5]:318-22.

[10]. Brannigan J, Tirodimos I, Zhang QY, Dowson C, Spratt B. Insertion of an extra amino acid is the main cause of the low affinity of penicillinbinding protein 2 in penicillin-resistant strains of Neisseria gonorrhoeae. Molecular microbiology. 1990;4[6]:913-9.

[11]. Lindberg $R$, Fredlund $H$, Nicholas $R$, Unemo M. Neisseria gonorrhoeae isolates with reduced susceptibility to cefixime and ceftriaxone: association with genetic polymorphisms in penA, mtrR, porB1b, and ponA. Antimicrobial agents and chemotherapy. 2007;51[6]:2117-22.

[12]. Tanaka M, Nakayama H, Huruya K, Konomi I, Irie S, Kanayama $A$, et al. Analysis of mutations within multiple genes associated with resistance in a clinical isolate of $<$ i> Neisseria gonorrhoeae $</ i>$ with reduced ceftriaxone susceptibility that shows a multidrug-resistant phenotype. International journal of antimicrobial agents. 2006;27[1]:206.

[13]. Hagman KE, Pan W, Spratt BG, Balthazar JT, Judd RC, Shafer WM. Resistance of Neisseria gonorrhoeae to antimicrobial hydrophobic agents is modulated by the mtrRCDE efflux system. Microbiology. 1995;141[3]:611-22.

[14]. Ameyama S, Onodera S, Takahata M, Minami $\mathrm{S}$, Maki N, Endo K, et al. Mosaic-like structure of penicillin-binding protein 2 gene [penA] in clinical isolates of Neisseria gonorrhoeae with reduced susceptibility to cefixime. Antimicrobial agents and chemotherapy. 2002;46[12]:3744-9.

[15]. Spratt BG. Hybrid penicillin-binding proteins in penicillin-resistant strains of Neisseria gonorrhoeae. 1988.

[16]. Yokoi S, Deguchi T, Ozawa T, Yasuda M, Ito S-i, Kubota $Y$, et al. Threat to cefixime treatment for gonorrhea. Emerging infectious diseases. 2007;13[8]:1275.

[17]. Unemo M, Golparian D, Syversen G, Vestrheim D, Moi H. Two cases of verified clinical failures using internationally recommended first-line cefixime for gonorrhoea treatment, Norway, 2010. Microscopy. 2010;120:A121N.

[18]. Ison C, Hussey J, Sankar K, Evans J, Alexander S. Gonorrhoea treatment failures to cefixime and azithromycin in England, 2010. Vol 16| Weekly issue 14| 7 April 2011. 2011:7.

[19]. Ohnishi M, Golparian D, Shimuta K, Saika T, Hoshina S, Iwasaku K, et al. Is Neisseria gonorrhoeae initiating a future era of untreatable gonorrhea?: detailed characterization of the first strain with highlevel resistance to ceftriaxone. Antimicrobial agents and chemotherapy. 2011;55[7]:353845.

[20]. Røttingen J-A, Cameron DW, Garnett GP. A systematic review of the epidemiologic interactions between classic sexually transmitted diseases and HIV: how much really is known? Sexually transmitted diseases. 2001;28[10]:579-97.

[21]. Unemo M, Golparian D, Stary A, Eigentler A. First Neisseria gonorrhoeae strain with resistance to cefixime causing gonorrhoea treatment failure in Austria, 2011. Euro Surveill. 2011;16[43]:pii= 19998.

[22]. Perilla M, Ajello G, Bopp C, Elliott J, Facklam R. Manual for the laboratory identification and antimicrobial susceptibility testing of bacterial pathogens of public health importance in the developing world. Haemophilus influenzae Neisseria meningitidis Streptococcus pneumoniae Neisseria gonorrhoeae Salmonella serotype Typhi Shigella and Vibrio cholerae. 2003.

[23]. Morency P, Dubois M, Gresenguet G, Frost E, Masse B, Deslandes S, et al. Aetiology of urethral discharge in Bangui, Central African Republic. Sexually transmitted infections. 2001;77[2]:125-9.

[24]. Rwahwir MP. Prevalence .socio-Demographic Factors and Antimicrobial Susceptibility of Neisseria gonorrhoea among men presenting with Urethral dischage at Mulago and Murchison bay Hospitals 2009. 